IOS Press

\title{
Editorial
}

\section{The new manuscript review system for the Semantic Web journal}

\author{
Pascal Hitzler ${ }^{\mathrm{a}}$, Krzysztof Janowicz ${ }^{\mathrm{b}}$ and Kunal Sengupta ${ }^{\mathrm{a}}$ \\ ${ }^{a}$ Kno.e.sis Center, Wright State University, USA \\ ${ }^{\mathrm{b}}$ University of California, Santa Barbara, USA
}

The Semantic Web journal relies on a non-standard review process which in particular features open and transparent reviews and encourages reviewers to disclose their identities [1,2]. While the continued success of the Semantic Web journal shows that this setup works in practice and is suitable for a high-quality journal, the overhead caused by the non-standard process has been significant, in particular for the editors and the editors-in-chief.

The main reason for this overhead was the unavailability of suitable manuscript review systems, as existing systems support only standard processes and are merely customizable within rigid and conventional bounds. For more than two years we thus had a rather cumbersome setup using, on the one hand, a commercial journal review management system, and on the other hand a public Website [2]. As a result, we had to constantly copy information from the review management system to the Website, and in fact we have done so manually. It was clear from the beginning that this was not going to be a solution which is sustainable over the long run, and so it was necessary to set up our own manuscript review system, tailored to the specific needs of the journal. In particular, we needed a tight integration of the review system with the journal's website, so that it would no longer be necessary to handle two independent systems.

The new review system went online in October 2012, and it has since served us very well. It is realized in Drupal, the popular Web Content Management System which underlies the Semantic Web journal website. Visitors of the website may in fact hardly notice a difference. However, authors who want to submit papers do so directly via the journal's website, rather than through a separate submission site. Likewise, reviewers and editors interact directly with the website.

While we are very pleased with the improvements resulting from the custom system, we will continue to develop it, and are happy about any feedback or comments we may receive. For 2013 we plan to embed Semantic Web technologies and thus support new types of queries over the journal's dataset.

In this issue of the journal, we present three papers. The first two are very substantial surveys of current research topics, and they continue our series of highly selective and well-received high-quality surveys published in the journal. The third article is a vision paper which argues for a systematic investigation of order (in the mathematical sense) for reasoning in the Big Data age. While we usually do not publish vision papers, we are happy to include this article of exceptional quality. It was fully reviewed to the standards of the journal.

\section{Acknowledgements}

Work on the new review system was supported by the State of Ohio Research Incentive grant Kno.e.CoM - Knowledge-enabled Content Management and by IOS Press. Kunal Sengupta acknowledges the hospitality of the University of California, Santa Barbara, during a visit while working on the system.

\section{References}

[1] P. Hitzler and K. Janowicz, Semantic Web - Interoperability, usability, applicability, Semantic Web 1(1-2) (2010), 1-2.

[2] K. Janowicz and P. Hitzler, Open and transparent: The review process of the Semantic Web journal, Learned Publishing 25(1) (2012), 48-55. 